\title{
Tromboembolismo pulmonar secundario a mixoma gigante de aurícula derecha
}

\author{
B. DAGA CALEJERO, M. GONZÁLEZ CARRETERO, R. ORTAS NADAL, \\ I. FERREIRA MONTERO
}

Servicio de Cardiología. Hospital Clínico Universitario Lozano Blesa. Zaragoza

PULMONARY EMBOLISM AND MYXOMA OF RIGHT ATRIAL

\begin{abstract}
RESUMEN
Se presenta el caso de un paciente diagnosticado de tromboembolismo pulmonar, en el que el ecocardiograma transtorácico demostró la existencia de una masa de gran tamaño en aurícula derecha. Se procedió a la extirpación completa de la misma, demostrando el estudio histológico un mixoma extensamente calcificado. El interés del caso se centra en la rareza de la localización y sobre todo extensión del mixoma, su aspecto macroscópico y microscópico atípicos y el hecho de que su diagnóstico y tratamiento evitaron la repetición de nuevos tromboembolismos pulmonares que podían haber sido letales.
\end{abstract}

PALABRAS CLAVE: Mixoma. Aurícula derecha. Tromboembolismo pulmonar.

\begin{abstract}
We describe the case of a patient diagnosticated of pulmonary embolism, in whom transthoracic echocardiography demostrated a large mass. It was completaly removed and histologic examination showed a very calcification myxoma. This case is of interest because localitation and extension of myxoma, the atypical histologic appearance, and in fact the diagnosis and treatment prevented new pulmonary embolism that could be lethal.
\end{abstract}

KEY WORDS: Myxoma. Right atrial. Pulmonary embolism.

Daga Calejero B, González Carretero M, Ortas Nadal R, Ferreira Montero I. Tromboembolismo pulmonar secundario a mixoma gigante de aurícula derecha. An Med Interna (Madrid) 2005; 22: 478-480.

\section{INTRODUCCIÓN}

Los tumores cardiacos primarios son poco frecuentes, su incidencia varía entre el 0,0017 y el 0,003\% (1). Aproximádamente, el $75 \%$ son benignos y de éstos más del $50 \%$ son mixomas (2). Así pues, el mixoma es el tumor cardiaco más frecuente en el adulto, caracterizado por tener una predilección por el sexo femenino, que suele diagnosticarse en edades comprendidas entre la tercera y sexta décadas (3), aunque se han descrito casos en fases extremas de la vida.

\section{CASO APORTADO}

Varón de 45 años de edad, con antecedentes personales de tabaquismo, criterios clínicos de bronquitis crónica y hábito enólico moderado, acude a urgencias por dolor en hemitórax derecho de características pleuríticas, sin otra sintomatología asociada. En la radiografía de tórax se objetivó una imagen sugerente de condensa- ción basal y, ante la sospecha de neumonía basal derecha, se instauró tratamiento antibiótico y analgésico ambulatorio.

Un mes después, el paciente seguía con dolor en costado derecho, junto con expectoración hemoptoica y astenia generalizada, motivo por el cual se decidió ingreso hospitalario. La exploración física mostró una hipoventilación en base derecha, sin fiebre ni disnea. El paciente se encontraba en ritmo sinusal y sólo destacaba en el electrocardiograma la presencia de un bloqueo incompleto de la rama derecha del haz de His. En el estudio hematológico y bioquímico sanguíneo se objetivó una leucocitosis con neutrofilia (Leucos: 19.800/mm3 con $85 \%$ de neutrófilos), resto de parámetros dentro de los límites de la normalidad. La gasometría mostró una ligera hipoxemia (pO2: $68 \mathrm{mmHg}$ ) con normocapnia y sin alteraciones en el equilibrio ácido-base. El D-dímero fue de $1.460 \mathrm{U} / \mathrm{l}$. Una nueva radiografía de tórax demostró una imagen de condensación pulmonar basal derecha con pérdida de volumen en hemitórax derecho y participación pleural. Con la sospecha de infarto, o neoformación pulmonar, se solicitó la realización de un TAC torácico que evidenció la existencia de una imagen hiperdensa en aurícula derecha y raíz de cava inferior, imagen sugestiva de infarto en lóbulo pulmonar medio

\footnotetext{
Trabajo aceptado: 6 de mayo de 2005
} 


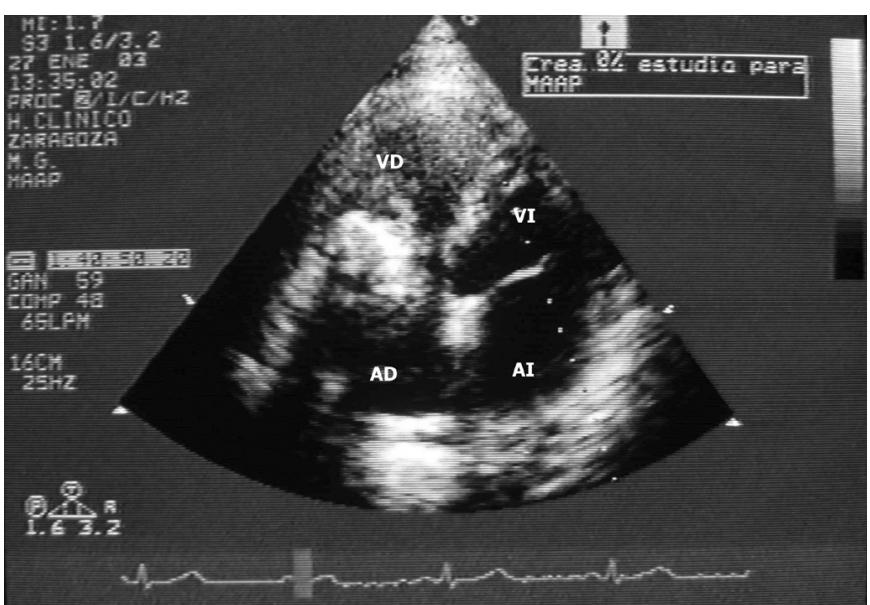

Fig. 1. Ecocardiograma transtorácico. Plano apical de 4 cámaras. Imagen polimórfica en aurícula derecha. AD: aurícula derecha. VD: ventrículo derecho. Al: aurícula izquierda. VI: ventrículo izquierdo. derecho y posible trombosis a nivel de arteria pulmonar derecha. La angiografía pulmonar mostró un tromboembolismo pulmonar inferior derecho con presiones pulmonares arteriales en los límites altos de la normalidad (Fig. 1).

Se solicitó un ecocardiograma, primero transtorácico y después transesofágico, donde se vio una imagen de 45 x $45 \mathrm{~mm}$, situada en aurícula derecha (Fig. 2), móvil, con densidad heterogénea e implantación amplia (30 x $40 \mathrm{~mm}$ ) en pared libre auricular, que hizo sospechar la existencia de un mixoma atípico.

El paciente, finalmente, se trasladó al Servicio de Cirugía Cardíaca del hospital de referencia donde fue intervenido mediante esternotomía media y bajo circulación extracorpórea, con resección total de un tumor de $30 \times 50 \mathrm{~mm}$ de diámetro, de aspecto infiltrante, implantado en cara anterior y lateral de aurícula derecha, que respetaba septo interauricular y aparato valvular tricuspídeo. Fue necesario la resección total de la pared libre auricular, colocando parche de pericardio autólogo para su cierre. Ante la sospecha de malignidad, se solicitó una biopsia intraoperatoria que no demostró signos de atipia celular. El estudio anatomopatológico definitivo evidenció la existencia de un mixoma cardíaco extensamente calcificado y con escasa matriz mixoide. El postoperatorio cursó sin complicaciones y

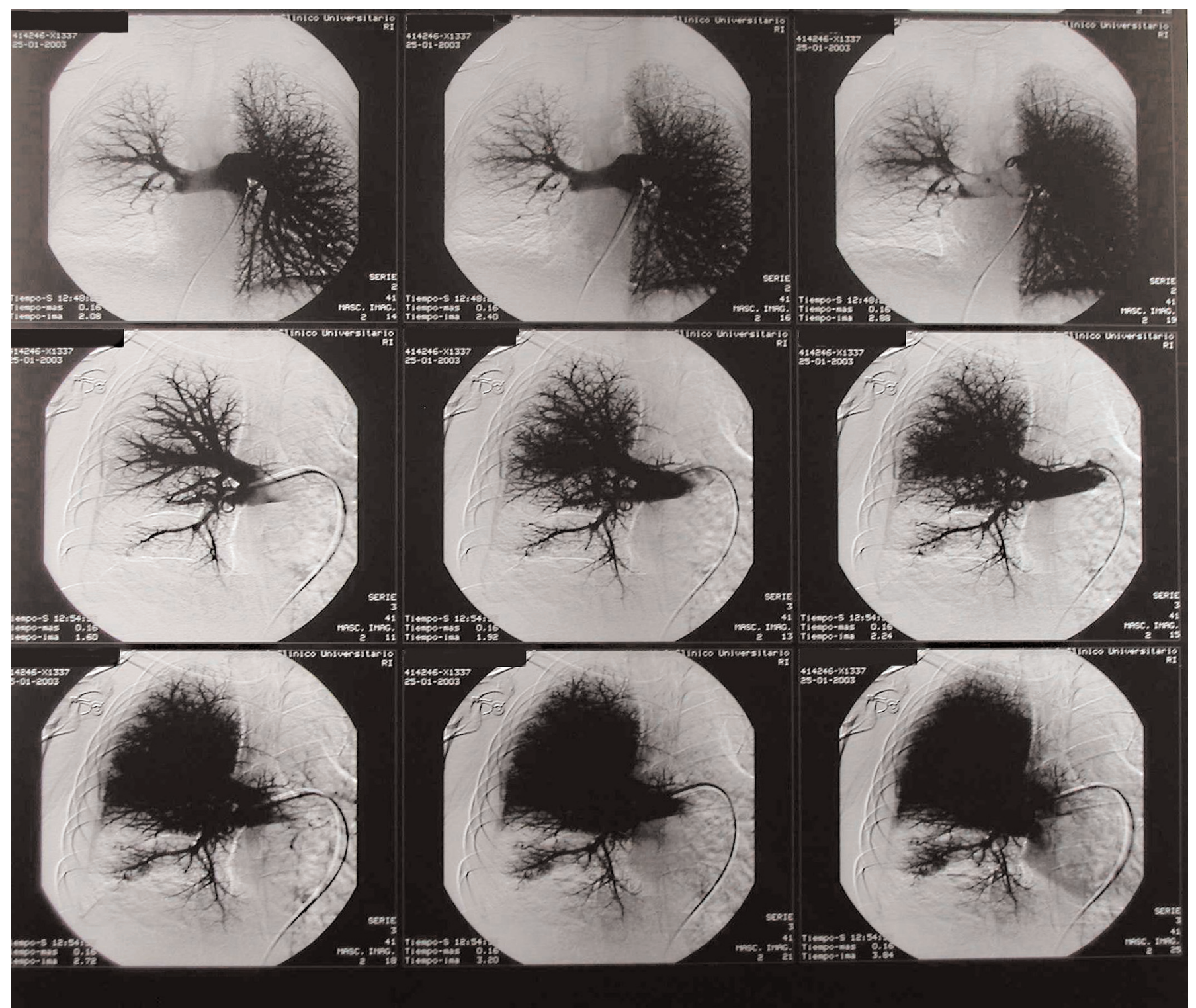

Fig. 2. Angiografía pulmonar donde se aprecia un defecto de perfusión en lóbulo inferior derecho. 
el paciente fue dado de alta a los trece días. En el último ecocardiograma de control no se ha evidenciado recidiva.

\section{DISCUSIÓN}

Las tres cuartas partes de los mixomas se localizan en aurícula izquierda, principalmente en el septo interauricular a nivel de la fosa oval, entre el 15 y el $20 \%$ se originan en aurícula derecha, siendo la localización ventricular, valvular o multifocal poco frecuentes (4). En nuestro caso el tumor asentaba en toda la pared anterior y lateral de la aurícula derecha, respetando el septum y la válvula tricúspide.

Macroscópicamente estos tumores suelen ser polipoides, pediculados, raramente sesiles, de consistencia blanda y gelatinosa, de tamaño variable, habitualmente entre 5 y $6 \mathrm{~cm}$ (5). Histológicamente se caracterizan por tener una matriz mixoide, con células poligonales típicas con un citoplasma eosinofílico. En tan sólo un $10 \%$ de los casos se observan focos de calcificación. En el caso referido, la pared de la aurícula derecha, incluida la orejuela, estaba engrosada y endurecida, con aspecto infiltrado, lo que inicialmente hizo sospechar una naturaleza maligna del tumor. Tras auriculotomía derecha se observó un tumor dependiente de toda la pared libre de la misma que protuía hacia la cavidad ventricular, a través del orificio valvular. Por otra parte, el estudio microscópico de la pieza demostró un área central muy calcificada con tejido tumoral únicamente en periferia.

\section{Bibliografía}

1. Blondeau PH. Primary cardiac tumours. French studies of 533 cases. Thorac Cardiovasc Surg 1990; 38: 192-6.

2. Abad C. Tumores cardiacos (I). Generalidades. Tumores primitivos benignos. Rev Esp Cardiol 1998; 51: 10-20

3. Salcedo EE, White RD, Cohen GI, Davison MB. Cardiac tumors: diagnosis and management. Curr Probl Cardiol 1992; 17: 73-137.

4. Hall RJ, Cooley DA, McAllister HA, Fraizer OH.. Neoplastic heart diseases. En: Alexander RW, Schalnt RC, Fuster V, editores. Hurst's the heart. McGraw-Hill Inc., 1998. p. 2295-318.

5. Hall RJ, Cooley DA, McAllister HA Jr, Frazier OH. Neoplastic heart
Las manifestaciones clínicas más frecuentes de estas neoformaciones incluyen síndrome constitucional, así como síntomas derivados de fenómenos embólicos y de la obstrucción al flujo intracavitario (6). Es conocida la capacidad de los mixomas para simular semiología de enfermedades sistémicas como síndromes polimiálgicos (7).

Nuestro paciente presentó síntomas y signos compatibles con un tromboembolismo pulmonar (TEP). En el estudio diagnóstico del mismo se solicitó la realización de un ecocardiograma, prueba que no sólo sirvió para determinar la fuente embolígena y confirmar la enfermedad tumoral, sino que también permitió valorar la inexistencia de repercusión hemodinámica grave.

El tratamiento de elección en este tipo de tumores es la extirpación quirúrgica8. Ésta debe llevarse a cabo con mayor celeridad en los pacientes con antecedentes de embolismo sistémico o pulmonar, como era nuestro caso, episodios sincopales o evidencia ecocardiográfica de masas multilobulares.

En conclusión, presentamos el caso de un mixoma de localización y extensión excepcional, toda la aurícula derecha excepto septo interauricular, con un aspecto macroscópico y microscópico atípicos, de aspecto infiltrado y extensamente calcificado, que se manifestó clínicamente como un tromboembolísmo pulmonar, en el que el ecocardiograma jugó un papel crucial tanto para el diagnóstico de la enfermedad primaria, como para el seguimiento de posibles recidivas. disease. In: Hurst JW, ed. The heart, arteries and veins. 7th ed. New York: McGraw-Hill, 1990: 1382-1403.

6. Markel M, Waller B, Armstrong W. Cardiac myxoma. A review. Medicine (Baltimore) 1987; 66: 114-25.

7. Gómez N, Vilar M, Ferreiro JL, Ibáñez J, Trinidad C, Noriega F. Síndrome polimiálgico y mixoma auricular. An Med Interna (Madrid) 1998; 15 : 370-372.

8. Lie JT. The identity and histogenesis of cardiac myxomas. A controversy put to rest [editorial]. Arch Pathol Lab Med 1989; 113: 724-6. 